\title{
ASSEMBLY LINE DESIGN PRINCIPLES USING SIX SIGMA AND SIMULATION
}

\author{
Benny Tjahjono \\ Peter Ball \\ John Ladbrook \\ John Kay \\ Manufacturing Department \\ Cranfield University \\ Cranfield, Bedford MK43 0AL, UK
}

\begin{abstract}
Many variables and constraints must be taken into account when designing a manufacturing facility such as an assembly line, which often depends on common practices and experience of the manufacturing engineers. Six Sigma has shown its benefits particularly in the process improvement and product development. This paper explores the applicability of Six Sigma and simulation techniques to derive a set of principles that can be used by manufacturing engineers to design assembly lines. The idea is to use simulation models as a basis for experimentation of parameters that are critical to the productivity of the lines. The sensitivity of these parameters were analyzed and the results from the experiments were then collated into a set of design principles that can be used as part of the facility design process.
\end{abstract}

\section{INTRODUCTION}

New product introduction typically involves the design of new manufacturing facilities such as machining and assembly lines. Time to market is therefore not only affected by the pace of product development but also the pace to design the manufacturing facilities. In the case of an assembly line, due to the complexity, stochastic nature and interconnectedness between machines and other components within the manufacturing systems, predicting the likely performance of the systems without tools such as computer-based simulation is difficult (Baines and Kay 2002).

The company where this study is based has used simulation as a decision making tool particularly in designing and installing its assembly lines worldwide. When a new line is built (or rebuilt), the layout is first drawn by the manufacturing process engineers. Many of the decisions, for examples buffer sizes, length of the conveyors etc, are made based on common practices and the experience of the process engineers. Once drafted, the layout is then passed onto the productivity engineers who will verify the design using simulation. The simulation study usually highlights a number of issues with the line and these are fed back to the process engineers. On many occasions, the proposed amendments can be implemented but it is not uncommon that the layout will need to be redesigned. This process is repeated until a feasible solution is agreed.

Six Sigma techniques have been extensively used in process improvement and product design. Design for Six Sigma (DFSS), for example, aims to prevent problems caused by poor design from occurring at the first place (Stamatis 2004). In this instance, DFSS will proactively reduce and, whenever possible, eliminate defects by imposing on a robust design process and focusing on the critical design parameters. The research project described in this paper explores the applicability of DFSS as a methodology to derive a set of principles that can be used to design assembly lines of car engines. It is envisaged that by better understanding the critical parameters of the line, a robust and efficient design process can be achieved because the iteration between the process engineers and the productivity engineers can be significantly reduced. In this project, simulation models have been used as a tool to enable experimentation and what-if scenarios. The models have been validated and are a true representation of the actual lines.

The paper first discusses the assembly line upon which the research project is based and the simulation tools used to develop the models. This is then followed by the methodology adopted in the project. The main body of research is presented in sequence following one of the DFSS approaches, i.e. DCOV (Design, Characterized, Optimize and Verify). The paper concludes with the detail account of the findings and lesson learnt from the project. 
Assembly lines are commonly used in a mass production system. An assembly line typically consists of a number of workstations arranged in a sequence and linked by a conveyor. The workstations can be automated, e.g. performed by robots, or manual where the work is carried out by operators, or even semi-automated, where an operation is carried out by a machine but maneuvered by an operator. In order to facilitate the analysis of the line, the assembly line is usually divided into a number of zones, e.g. zones A to L in Figure 1(a).

In a typical engine plant, car engines are mounted on specialized jigs called platens and transported along the conveyors from one workstation to another. The platens can be rotated allowing the operators to access different parts of the engines and to perform different operations. At each workstation, a task has to be completed according to a standardized processing time, also known as the cycle time. When an operator is working on that particular engine, the conveyor is usually stopped and remains stationary until the task is completed. The operator will then release the engine to the next workstation. This process is repeated along the assembly line and the whole sequence of these operations leads to the production of a complete car engine.

In the case of manual assembly, an operator sometimes need more time to complete the task causing starvation at the next workstation, or conversely, is quicker than the standardized cycle time, causing bottleneck at the next workstation. For this reason, two workstations are often decoupled by a buffer to ensure the balance of the line and smooth flow of the engines. In the case of car assembly lines with conveyor belts, the size of the buffer is therefore determined by the length of the conveyor (or the space) between the two workstations.

The simulation tool used in this project is a proprietary Excel spreadsheet that acts as an input/output interface to Witness. The spreadsheet has been significantly enhanced with many features, one of which being the automatic generation of simulation codes. The use of such an interface can significantly speed up model building, making it easier for users who are not expert in simulation to build and modify complex assembly lines models consisting of combination of machines and operators who work to assembly the car engines (Benedettini and Tjahjono 2009). The automatic code generation has been made possible by programming macros inside Excel that link to a library of custom designed Witness modules. The library of modules consists of the equipment typically present on an engine assembly line, e.g. manual/automatic workstations, conveyors, turntables etc. Figure 1(b) shows the screenshot of the simulation tool where the cycle time has been transferred to the corresponding Witness model.

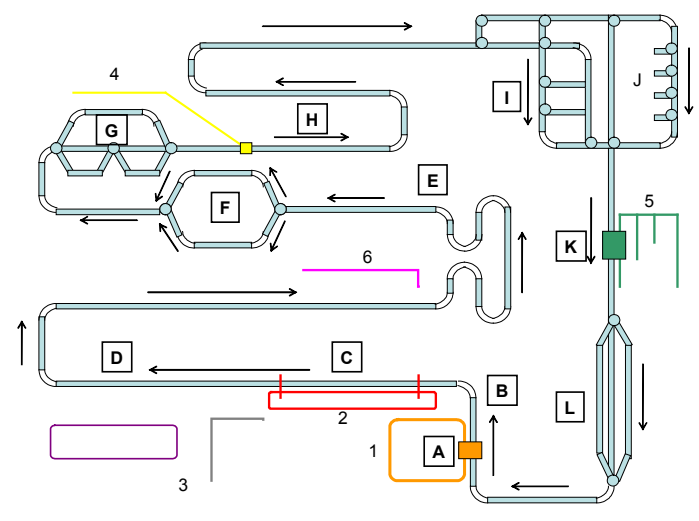

(a)

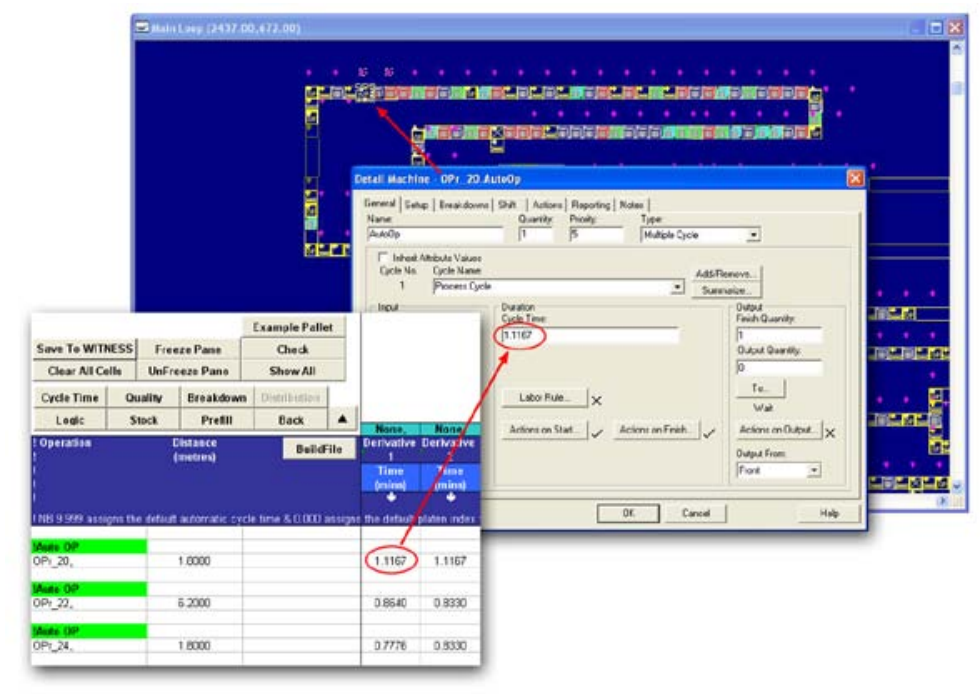

(b)

Figure 1: The assembly line and simulation tool used

\section{METHODOLOGY}

The overall aim of this project is to provide a set of principles that can be used to assist manufacturing engineers in the design of the assembly lines in order to maximize their productivity. The design process is inherently a complex task affected by many factors, and as such, identification of critical parameters, variables and related metrics is necessary to set the boundary of the project. Rather than relying on common practices, the identification of these parameters uses a data-driven process which ensures the robustness of the design process, and eventually will shorten the time to market. For this reason, DFSS is chosen as the overarching methodology for the research project and is implemented in conjunction with simulation. 
DFSS is a proven method that aims to improve process effectiveness while focusing on predicting and improving quality before product and processes are launched (Banuelas and Antony 2003). One of the approaches within DFSS called DCOV (Design, Characterized, Optimize and Verify) has been chosen primarily because of its suitability to the project and that the approach has already been used in many Six Sigma projects within the company. Figure 2 summarizes the main steps of DCOV approach used in the project and will be described in more detail in the following sections.

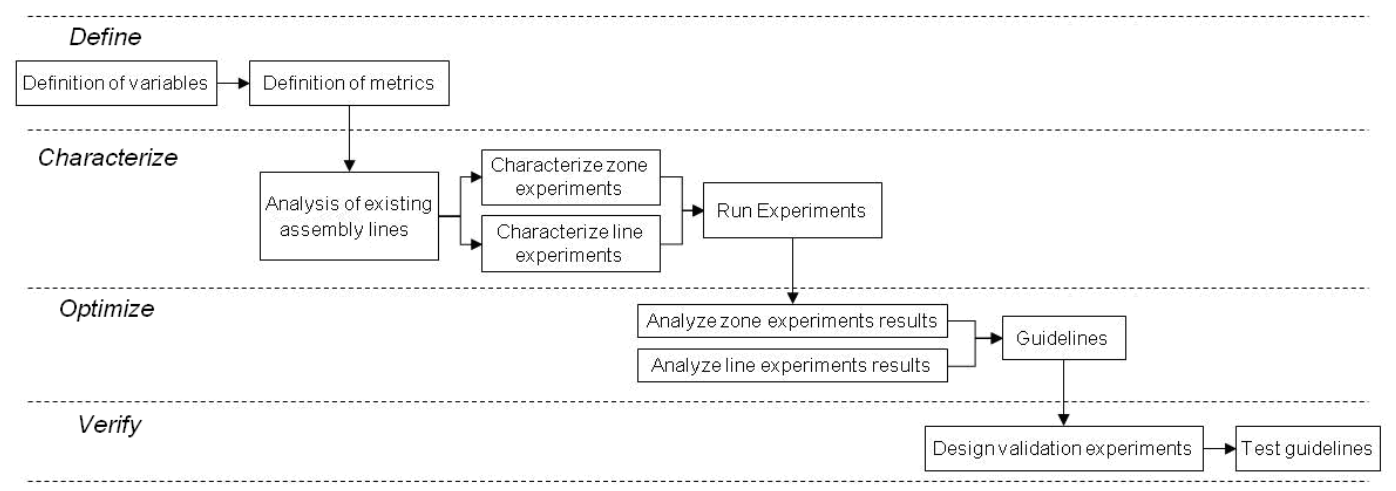

Figure 2: Research methodology

The experiments were carried out on the situation models that are a true representation of the actual lines from four engine plants. For confidentiality reasons and in order to have greater freedom to discuss the results and findings, the engine plants are referred to as plants A, B, C and D hereafter. Plants A and B are used for experimentation purposes to derive the design guidelines. The guidelines were then applied to plants $\mathrm{C}$ and $\mathrm{D}$ in order to test their validity.

\section{DEFINITION OF METRICS AND VARIABLES OF STUDY}

The metrics and the variables used in this project are denoted using transfer functions, which are the expressions that link the quality metrics of a process with the inputs that define and control this process. These types of expression are particularly useful in order to identify and communicate the main variables affecting the system.

The main quality metric considered in this project is efficiency, which can be defined as a measure of actual output relative to some standard of output (Reid and Sanders 2005). As the line is divided into a number of zones, (where each zone consists of several operations/workstations), the efficiency of the assembly line therefore depends on efficiency of the zones.

Zone efficiency is considered as a function of the efficiency of operators and machines, quality of operations, buffers and rework needed, and can be expressed by the following transfer function:

$$
Y_{1}=\eta_{\text {zone }}=f\left(\eta_{\text {operators }}, \eta_{\text {machines }}, Q_{\text {operations }}, B, R\right)
$$

where

$$
\begin{aligned}
& \eta_{\text {zone }}=\text { efficiency of a zone } \\
& \eta_{\text {operators }}=\text { efficiency of operators within a zone } \\
& \eta_{\text {machines }}=\text { efficiency of machines within a zone } \\
& Q_{\text {operations }}=\text { quality of operations } \\
& B=\text { size of buffers } \\
& R=\text { level of rework needed }
\end{aligned}
$$

The line efficiency is expressed as a function of the efficiencies of the entire zones:

where

$$
Y_{2}=\eta_{\text {line }}=f\left(\eta_{\text {zone }}\right)
$$

$$
\eta_{\text {line }}=\text { efficiency of a line }
$$


The design parameters that define the system's transfer function need to be further detailed and converted into a number of independent variables that may affect the performance of the line. In DFSS, this is done using the Critical to Quality (CTQ) tool. The parameters that have been identified as sensitive to the performance of the lines are: zone length, zone complexity, buffers and operator density. Buffers are further divided into two: within zones and between zones. Having identified the variables, the next step is to decide the relevant experiments in order to assess how these variables could impact the efficiency.

\section{CHARACTERIZATION OF EXPERIMENTS}

Characterizing the experiments involves analysis of the design parameters in the existing lines. Types of operation, cycle times, length of buffers, machines breakdown and operators' over-cycles are some of the simulation parameters recorded. This information provides a general idea of the existing practices in the design of assembly lines and a sense of the order of magnitude of the different parameters to be studied.

The analysis of the two assembly lines from engine plants A and B provides the necessary information in order to classify the different zones depending on the types of operations: either manual or automatic. The intention of such classification is to study the effect of type of operations on the design of the line (zone complexity) and the effect automatic machines have in the overall performance of the zones and lines.

Experiments are divided into two main subgroups: zone experiments and line experiments. Zone experiments have been further divided into three categories:

1. Experiments focusing on the distribution of buffers inside the zone.

2. Experiments focusing on the influence of cycle times.

3. Experiments on platens and breakdowns.

\subsection{Experiments carried out at the zone level}

Zone experiments analyze the behavior of a zone in isolation from other zones. These experiments have the boundary conditions to ensure that the zone under investigation never gets blocked nor starved of parts, conceptually implying the availability of an infinite buffer capacity at each end of the zone.

1. Even distribution of fixed amount of buffer spaces between all operations in the zone. The range of buffer spaces is from 0 to 8 spaces. It was hoped that it showed a tipping point (if throughput stabilized at a given buffer size and then decreased) or at least a point where throughput increase was not worth it compared to the increase in costs associated to the conveyor length (see Figure 3).

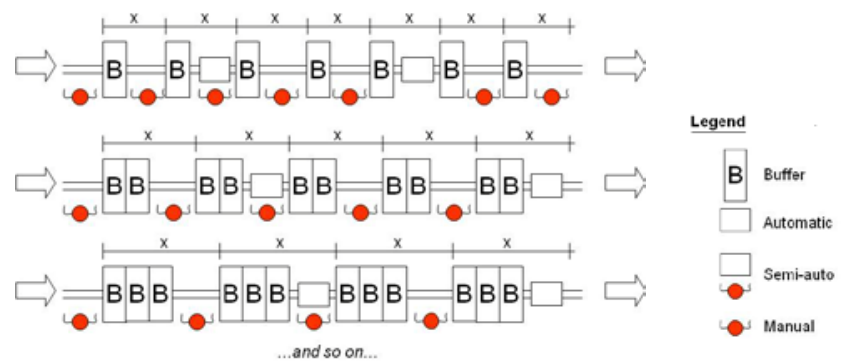

Figure 3: Even distribution of buffers

2. Bow-tie distribution. This is the distribution of a fixed amount of buffer spaces amongst all operations following a triangular shape, also known as a 'bow-tie'. The principle behind the bow-tie shape is to identify a bottleneck operation in a zone, and to assigned the significantly large buffer size before and after it. The operations both upstream and downstream will have relatively smaller buffer sizes further away from that bottleneck operation (see Figure 4).

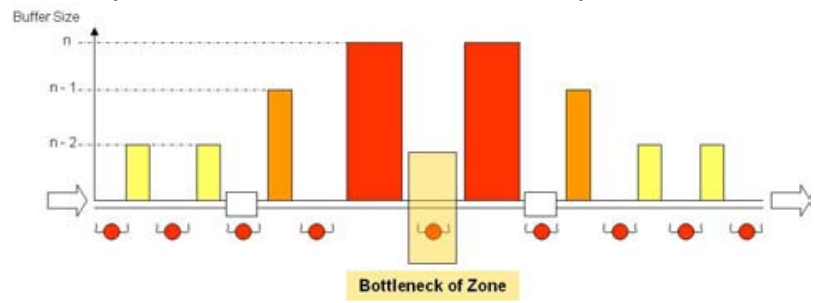

Figure 4: Bow-tie distribution of buffers 
3. Operation clustering. This experiment is carried out to cluster the operations by inserting buffer between clusters of operations as shown in Figure 5. The aim was to investigate whether operations can be grouped together without buffers in between those operations so that the performance of the line remains unaffected.

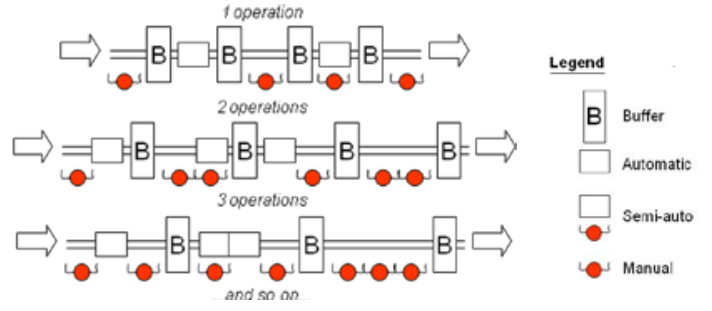

Figure 5: Operation clustering

4. Cycle times for manual and automatic operations. Automatic operations (i.e. typically performed by robots) are designed to work at a slightly higher speed than the manual operations. In other words, the speed of the machines will drive the productivity. The objective of this experiment is to measure the sensitivity of the line to changes in the cycle times of the automatic operations. This is done by fixing the cycle times of manual operations and varying the cycle times of automatic machines from $70 \%$ to $130 \%$ of the original cycle time in steps of $10 \%$. Performance and efficiency of the line is then recorded.

5. Breakdown. Although breakdown is outside the scope of this project, the purpose of this experiment was to check the results obtained on buffer utilization for the even distribution configuration. Three groups of experiments were carried out. In the first group, all the breakdowns were disabled in order to check the consistency of the results. The second group considered only the breakdown at the first operation and similarly the last experiment considered only the breakdown at the last operation (see Figure 6).

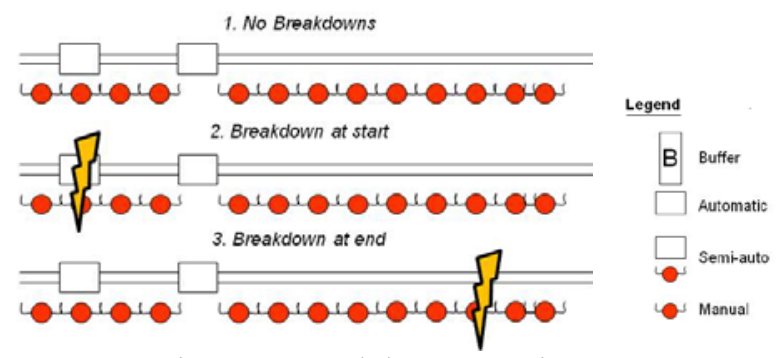

Figure 6: Breakdown experiments

\subsection{Experiments carried out at the line level}

As the efficiency of the line depends on the efficiency of the zones, a number of experiments were also carried out to investigate the effect of modifications at the zone level to overall performance of the line.

1. Even distribution of fixed amount of buffer spaces between all operations in the line. The lines have been configured with an even distribution of buffer spaces, ranging from zero to six. The aim of this is to validate the zone experiments regarding this buffer configuration and assess the relationship between the type of operations that each zone contained and their observed behavior in these conditions.

2. In zone bow-tie distribution. The bow-tie buffer shape is applied to all zones within the line and its performance is compared to that of the previous experiments. The purpose is to investigate the effect of in-zone buffer distribution on line performance (rather than just on the zone) and also provides additional data regarding the sensitivity of each of the zone to this distribution (see Figure 7). 
Tjahjono, Ball, Ladbrook and Kay

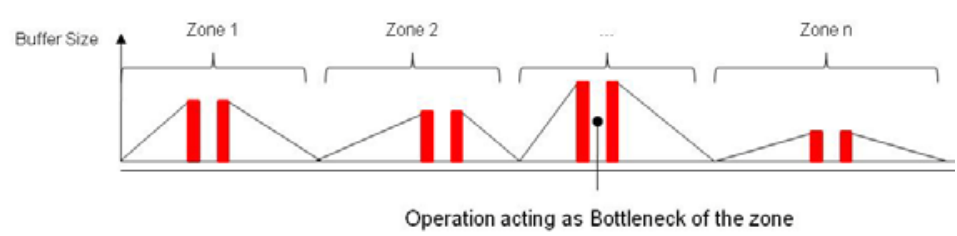

Figure 7: In zone bow-tie distribution

3. Between zone bow-tie distribution. The buffers between zones are shaped using the bow-tie principle but leaving the buffers inside the zones unchanged. The only buffers manipulated are the ones between zones (see Figure 8).

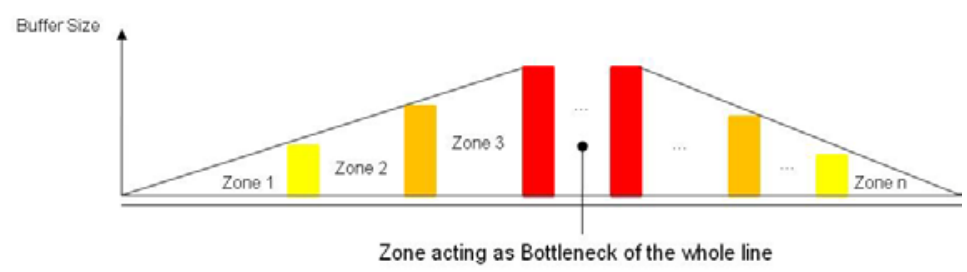

Figure 8: Between zone bow-tie distribution

4. Bow-tie distribution of cycle time for 1 and 2 buffer space. An experiment with cycle times was designed to test the behavior of the line when rebalancing zone cycle times. First, the bottleneck zone was identified and its operation cycle times were set to the slowest. That slowest cycle time will obviously dictate the pace of the whole line. Then a cycle time of $10 \%$ shorter than the bottleneck one is assigned to the zone further away from the bottleneck. The 'slope' of increased and decreased cycle times is kept constant. The whole line is then shaped using the bow-tie configuration, with each zone having increasingly longer cycle times as they are approaching the bottleneck zone. To keep these experiments compatible with the majority of the previous ones, the buffer distribution is kept even, using both 1 and 2 spaces between operations. The experiment is also repeated with a $20 \%$ decrease at the edge of the zone, instead of $10 \%$ respective to the bottleneck cycle time.

5. Variation on the number of platens. The experiments for even distribution of 3 buffers up to 6 buffers are repeated for both plants increasing the number of platens each time by $10 \%, 20 \%, 30 \%$ and $50 \%$ over the original figure. The objective of this experiment is to investigate whether the justify the fact that the decreased in productivity is due to an inadequate amount of platens and not because of the length of the line per se.

OPTIMIZATION AND ANALYSIS OF THE RESULTS

The data for productivity and buffer utilization for all experiments were then compiled into a spreadsheet. Data from both the zone and line experiments were analyzed separately. In many occasions, information about zone performance was also taken from the line experiments in order to back up or provide counterexamples against proposed trends.

The analysis was carried using the regression analysis. Some experiments were conducted in order to test out assumed causes for counter-intuitive results and to make sure a replication confirmed a given figure. In particular, the statistical study is also used to obtain a numerical value for the strength of the correlation amongst the parameters as well as to give a more precise indication of the level of inter-dependency of the compared variables.

To reach these final results, each type of experiment was analyzed and then the findings were compiled. If the evidence for a given trend is apparent in several of these experimental batches, it is considered worthy of being validated and added to the final set of findings.

\subsection{Even distribution of buffers}

The even distribution experiments showed that, taking cost into account, the best amount of buffers between operations is equivalent to buffer spaces. More than two buffer spaces give incrementally lower increase in throughput (or called Jobs per Hour - JPH) (Figure 9) 


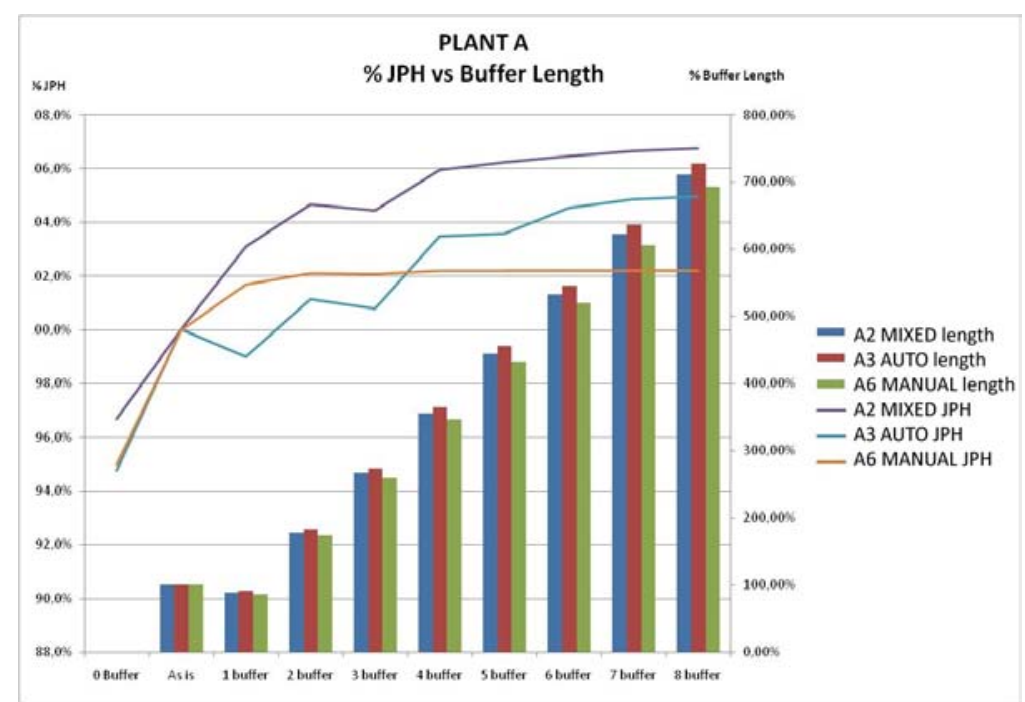

Figure 9: Plant A - JPH vs Buffer length

It is also apparent that for configurations that result in longer lines (spaces added), the performance of the line drops. An increase to up to $50 \%$ in the number of platens (which obviously needs additional investment), is needed to maintain the level of JPH. It is therefore demonstrated that the gaps in the line (due to the physical length but insufficient platens) reduced performance significantly (Figure 10).

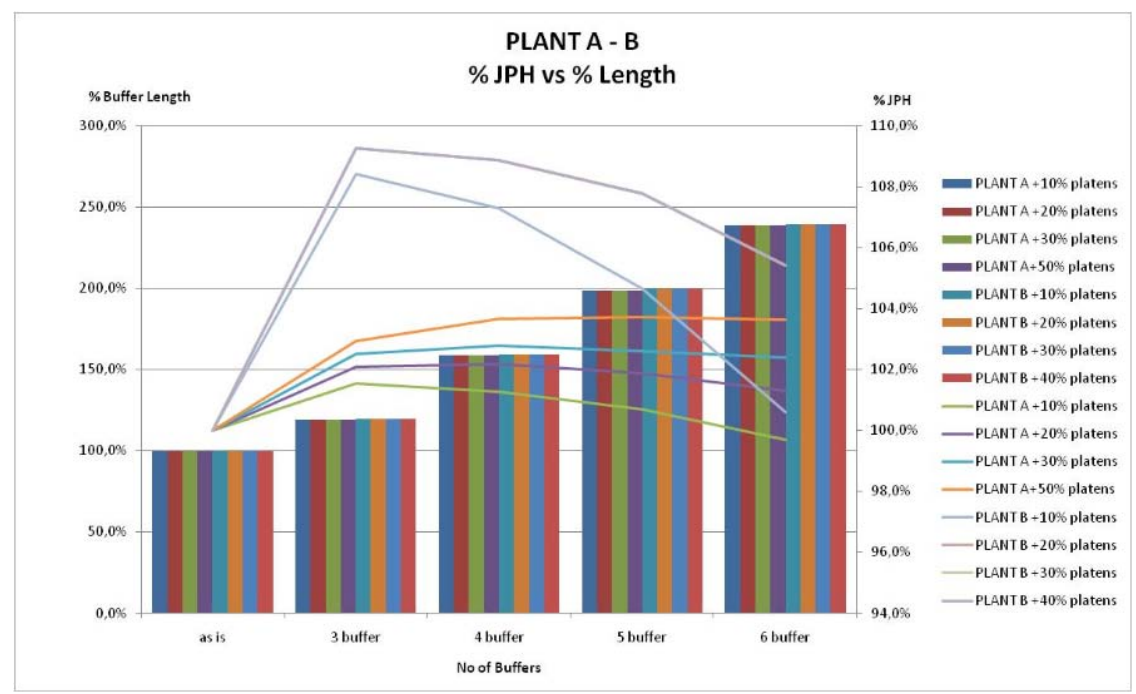

Figure 10: Plants A \& B - Platens analysis: JPH vs Buffer length

The preliminary principle is therefore to avoid having operations without buffers in between at any point in the line. Furthermore, the results have proved the necessity of increasing the amount of platens for lines which are significantly longer than the existing ones.

\subsection{Clustering of operations}

Clustering of operations proved to have a negative impact on performance of the zone, with all JPH figures dropping as cluster size increases. This fact was taken as evidence for the negative impact of zero space buffers in overall performance, since these experiments have the same buffer length as the even-two distribution and only differ in an increasing amount of operations without buffering between them. 


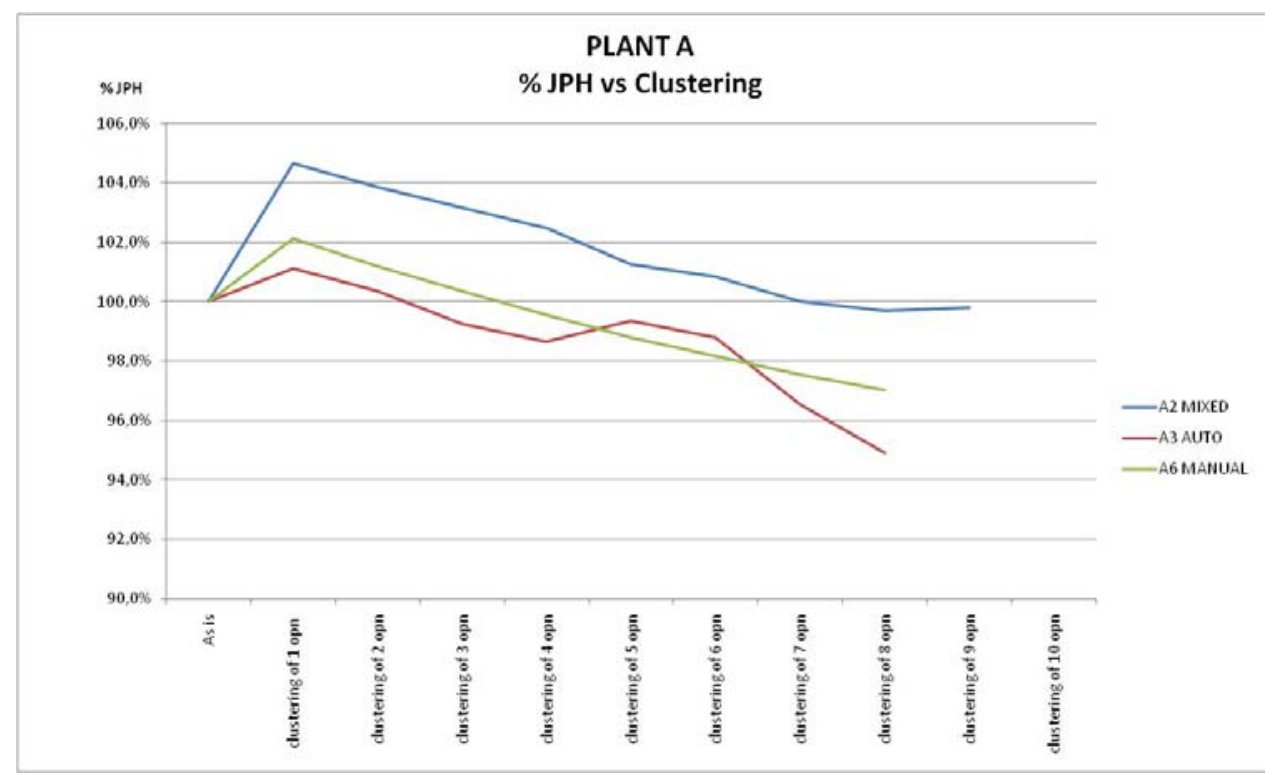

Figure 11: Plant A - JPH vs Operation clustering

\subsection{Bow tie}

The bow-tie distributions in general give better performance than the original configuration, but in most cases are worse than the even-two distribution for the same buffer length.

The second finding regarding this buffer configuration is the fact that in the zones, their performance has been among the best, but in the line experiments employing it, this tendency has not been shown. This is thought to be related to its varied results in different zones. The pace of the line will be dictated by its slowest zone and in some cases these configurations apparently reduce the efficiency of the line. Applying a bow-tie configuration to the whole line, therefore, may improve many zones. However, if it affects the bottleneck zone negatively then the overall performance will drop. Further experiments were carried out during the verification step to better understand these behaviors, both at the zone and the line levels.

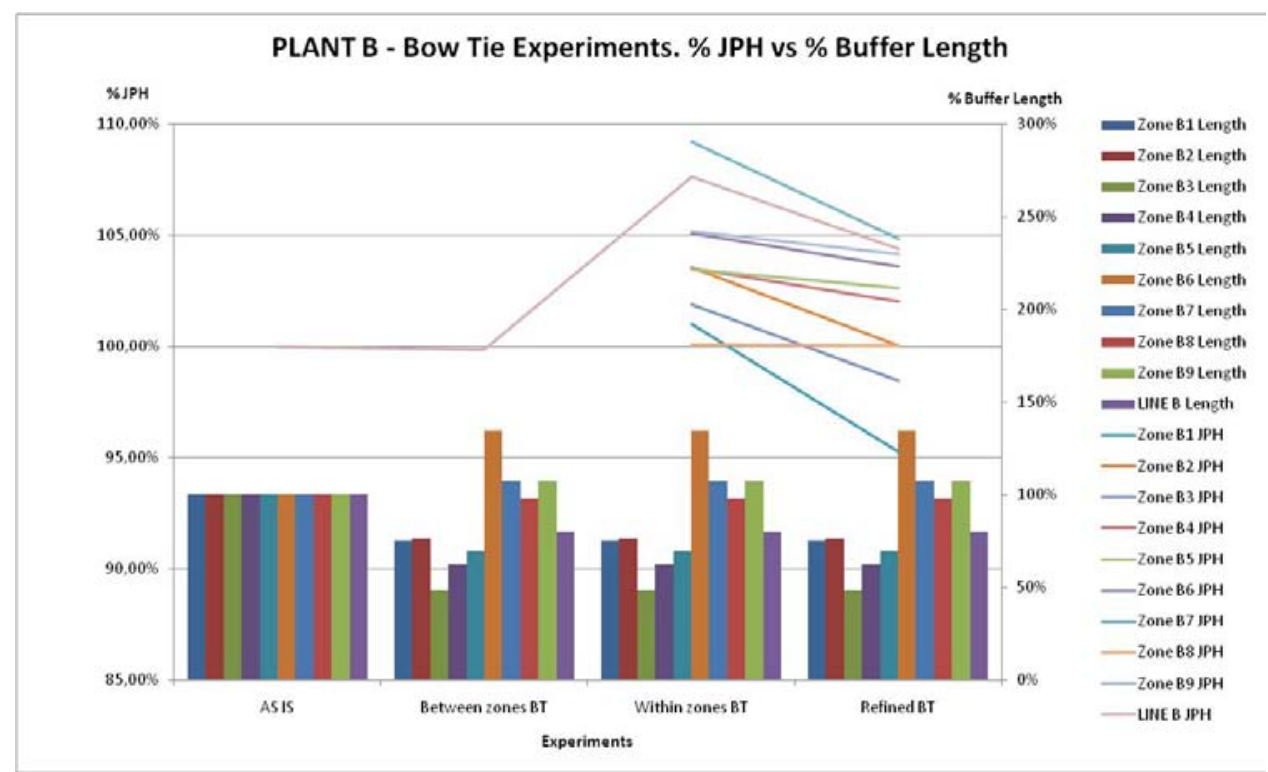

Figure 12: Bow-tie experiment - JPH vs Buffer length 


\subsection{Summary of findings}

Evidence showed that clustering operations (in groups of any size) reduced performance of both the zone and the line. It is therefore proposed as the fundamental guideline to avoid having 'zero buffer' between operations in any case. The zero buffer appears to be the inhibitor for the performance.

The second finding was that the bow-tie distribution of buffers within the zone applied to the whole line was found less useful. However, it was apparent that some zones benefited greatly from this configuration and therefore potential benefits could also be gained from expanding the experimentation to include this in more detail.

The third finding was the fact that the length of the line had to be proportional to the amount of platens to avoid gaps in the line. Bearing in mind that additional factors such as cycle time and machine/human breakdown are beyond the scope of this project, further study could be done to calculate the optimum amount of platens for any given line length.

Figure 13 illustrates some of the most important findings collected in a visual format for both plants analyzed.
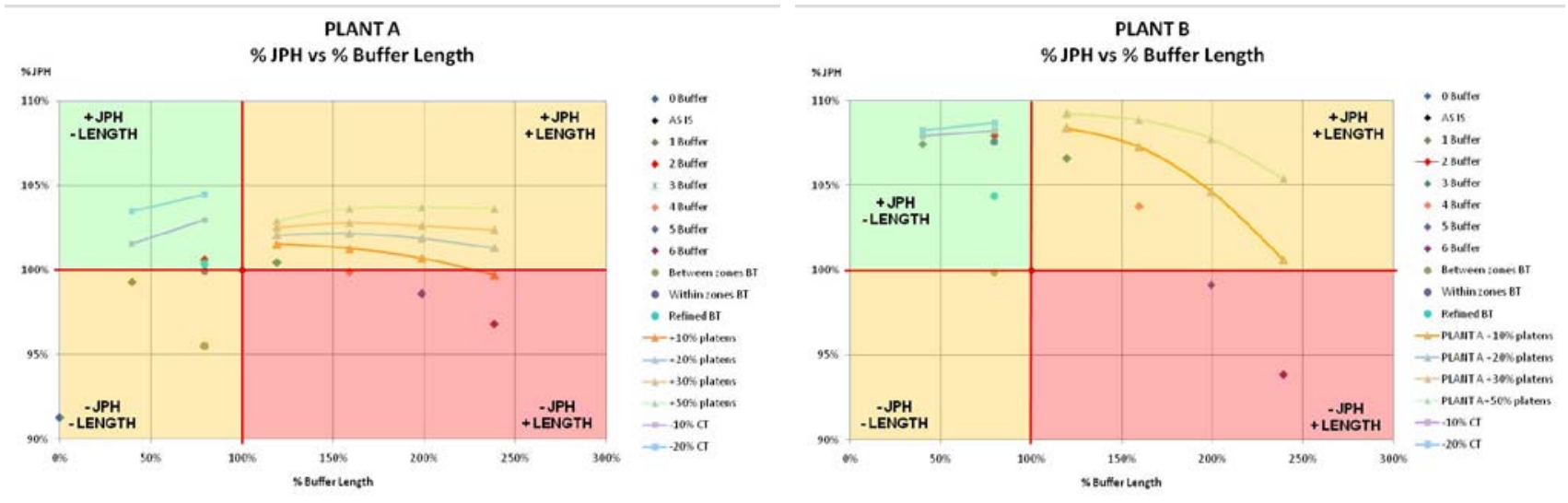

Figure 13: JPH vs Buffer Length

\section{VERIFICATION OF THE FINDINGS}

Having developed the preliminary guidelines, the next step in the project was to verify them. For the guidelines to be generic and not a product of the particular details of each line, they should produce reasonably similar results when used in lines that have not been studied before. Experiments at this phase therefore needed to be replicable. Figure 14 show the results obtained for the experiments in both plants $\mathrm{C}$ and $\mathrm{D}$. Results from this step, to a large extent, were coherent to the ones obtained during the Optimization phase.
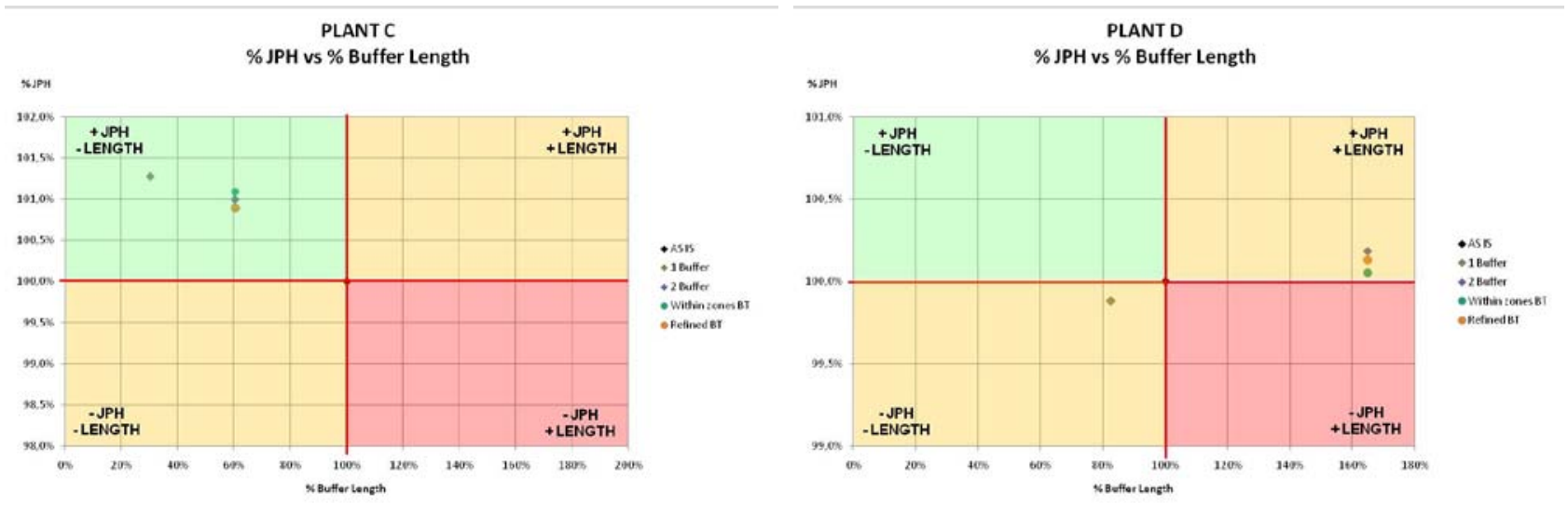

Figure 14: Verification - JPH vs Buffer length 
The results from the Verification phase were mixed but successful overall. Experiments run on plant $C$ have improved the original performance of the line in every case, with the best result being the even distribution of one buffer space, which performed better in terms of JPH and is $60 \%$ shorter than the original line.

Experiments on plant $\mathrm{D}$ were quite different. Those using the even distribution of two buffers length were all better in terms of JPH but for this, it required longer lines than the original. In this case, the one buffer even distribution was, again, the best choice since it only lost $0.01 \%$ of the original throughput, but was $20 \%$ shorter than the original line.

In both lines, the performance of both bow-tie configurations was almost the same as the two buffer even distribution, but the results varied since in each plant the JPH improvement for each of them was different. Regular bow-tie provided better results in plant $C$ than in plant $D$. Further research will be required to find out the reasons why these differences in behavior appeared. The results, nevertheless, provided evidence that there was potential in such configurations, but further experimentation is required in order to build upon these initial results.

\section{CONCLUDING REMARKS}

The main findings have been compiled into a mini guide that can be used by process engineers during the design process of a new assembly line.

\subsection{Findings}

The following findings have been summarized from the experiments:

- The bow-tie distributions cannot be recommended since there are insufficient data relative to their variations in performance from one line to another. Further study of the individual characteristics of the zones may help clarify this.

- The even distributions of buffer, which is a way to eliminate operation clusters (i.e. those which have zero buffers between them) has proven to be the important principle to follow when designing lines. The amount to choose, be it one or two, may vary but it seems clear that they represent a substantial improvement.

- Productivity drops as the length of the line increases. A shortage of platens may explain this behavior. If the line lengthens without increasing the number of platens, there are gaps in the line which reduce the overall performance.

\subsection{Benefits}

There are several benefits derived from this research project, some of which are listed below:

- The implementation of the best and most effective buffer configuration achieves the same level of JPH as in the existing assembly lines but decreases the buffer length and consequently the required space for its installation.

- The savings in buffer length should be directly reflected in a reduction of building costs for the facility. Shorter lines should require less investment in conveyors, land and smaller factories.

- The provision of guidelines in the form of a workbook will serve the design/process engineers as a roadmap for the design of future assembly lines; potentially reducing the planning time of these.

- A starting point to explore further the potential benefits that Six Sigma, or DFSS, can have for process design, opening a promising line for further research.

\section{ACKNOWLEDGMENTS}

The following individuals are part of the project team and their contribution to the work reported herein is gratefully acknowledged: Ms J Calleja, Mr. M Minguet, Mr L Narasimha, Mr J Nogueira, Mr A Rivas, Ms C Scorzafave, Mr A Srivastava, Mr S Srivastava and Mr A Yadav.

\section{REFERENCES}

Baines, T.S., and J.M. Kay. 2002. Human performance modelling as an aid in process of manufacturing system design: a pilot study, International Journal of Production Research 40: 2321-2334

Banuelas, R., and F. Antony. 2003. Going from six sigma to design for six sigma: an exploratory study using Analytic Hierarchy Process. The TQM Magazine 15: 334-344.

Benedettini, O., and B. Tjahjono. 2009. Towards an improved tool to facilitate simulation modelling of complex manufacturing systems. International Journal of Advanced Manufacturing Technology 43: 191-199

Reid, R., and N. Sanders. 2005. Operations Management: An Integrated Approach. John Wiley \& Sons.

Stamatis, D.H. 2003. Six Sigma Fundamentals: A Complete Guide to the System, Methods and Tools. Quality Press 


\section{AUTHOR BIOGRAPHIES}

BENNY TJAHJONO is the Director of Manufacturing Masters Program at Cranfield University. His research interest is in simulation modeling and analysis of manufacturing systems. He is currently leading a research team to work on the EPSRCfunded project entitled An Evolutionary Approach to Rapid Development of Simulation Models (grant no EP/E037631/1). Dr Tjahjono has been working closely with global companies, such as Rolls-Royce, Ford and Airbus, on several funded projects associated with the decision making process using discrete-event simulation.

PETER BALL is a Senior Lecturer in Manufacturing Operations. His research area covers manufacturing simulation, sustainable manufacturing, manufacturing supply design, supply chain design and e-business simulation and modeling. Research projects include the development of business collaboration models, business process outsourcing and development of ebusiness modeling as well as in business process innovation and brand value performance measurement.

JOHN LADBROOK is a visiting fellow at the Manufacturing Department. His MPhil degree from University of Birmingham concerns with modeling breakdown within manufacturing systems. His hands-on experience in manufacturing simulation dated back to early eighties when he first introduced the use of discrete-event simulation as part of decision making process in the company he worked for. His interest is in promoting the use of simulation by non-simulation expert, hence increasing the uptake of the technique within manufacturing companies.

JOHN KAY is a Professor of Manufacturing Systems Engineering and Head of the Manufacturing Department. He has over twenty years experience of working with industry to develop graduates into manufacturing professionals. He has undertaken around 150 projects with many companies including Rolls-Royce, Airbus, BAE Systems and many small enterprises. His research interests include manufacturing systems modeling, lean and agile manufacturing, group technology and cellular manufacturing. 